\title{
Synthesis of arylidene dihydropyrimidinones and thiones catalyzed by iron (III) phosphate
}

\author{
Fatemeh Moradi ${ }^{\mathrm{a}}$ and Farahnaz K. Behbahani ${ }^{\mathrm{a}^{*}}$
}

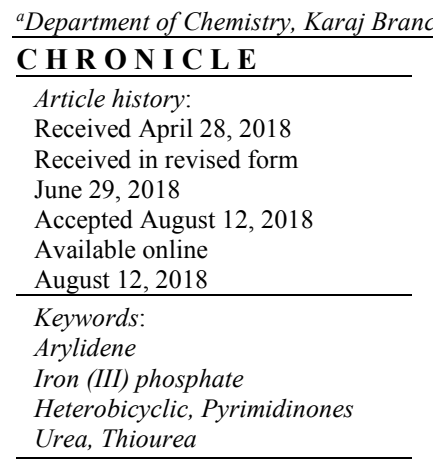

\section{Introduction}

Pyrimidinones are used in various pharmaceutical and biochemical fields. ${ }^{1,2}$ Therefore, an interest for the synthesis of 3,4-dihydropyrimidin-2(1H)-ones (DHPMs) and their derivations is tremendously increasing. ${ }^{3}$ One of the most important functionalized pyrimidinones is fused derivatives with an arylidene group. These heterocyclic compounds are significant intermediates for the preparation of many biologically active products. For example, some of them show a broad-spectrum antitumor activity. ${ }^{4}$

Because of the various therapeutic utility of arylidene heterobicyclicpyrimidinones, a number of synthesis routs for these derivatives were developed. ${ }^{5}$ In most cases, using strong Brönsted acid such as $\mathrm{HCl}$ or base such as sodium alkoxide or potassium hydroxide was necessary for the progress of the reaction. ${ }^{6,7}$ More useful procedures are three-component condensation with aromatic aldehydes, cyclopentanone, and urea or thiourea in presence of stoichiometric amounts of $\mathrm{TMSCl}^{8}$ and $\mathrm{YbCl}_{3}{ }^{9}$ Therefore, we wish to report the synthesis of arylidene heterobicyclicpyrimidinones and thiones using $\mathrm{FePO}_{4}{ }^{10,11}$ in the presence of arylaldehydes, cyclopentanone, and urea or thiourea. Moreover, this approach is known as an important economical and environmentally benign process in synthesis 
chemistry, because it decreases the number of reaction steps, energy consumption and waste (Scheme $1)$.

\section{Results and Discussion}

To optimize the reaction conditions, the reaction of benzaldehyde, cyclopentanone, and urea was selected as a model reaction in presence of catalytic amount of $\mathrm{FePO}_{4}$. The best result was obtained when the reaction was carried in a 2:1.1:1.2 mole ratio of benzaldehyde, cyclopentanone, and urea in the presence of $\mathrm{FePO}_{4}(20 \mathrm{~mol} \%)$ under solvent-free condition at $110{ }^{\circ} \mathrm{C}$ for $4.0 \mathrm{~h}$. In a catalyst free reaction, without $\mathrm{FePO}_{4}$, no desired product was obtained in $4.0 \mathrm{~h}$. But, very low yield (20\%) was resulted after $48 \mathrm{~h}$. To use these optimized conditions (benzaldehyde $(2 \mathrm{mmol})$, cyclopentanone $(1.1$ $\mathrm{mmol})$, urea $(1.2 \mathrm{mmol})$, and $\mathrm{FePO}_{4}(20 \mathrm{~mol} \%)$ under solvent-free condition at $110{ }^{\circ} \mathrm{C}$ for $4.0 \mathrm{~h}$.), the reaction between various aromatic aldehydes and cyclopentatone in presence of urea and thiourea was investigated (Table 1). It was found that all the reactions proceeded smoothly to give the corresponding arylidene heterobicyclicpyrimidinones and thiones in high yields. Both aromatic aldehydes bearing electron-donating and electron withdrawing groups gave excellent yields. The mildness of the procedure makes it very selective, as it tolerates a variety of functionalities, including; $\mathrm{N}(\mathrm{Me})_{2}, \mathrm{Cl}, \mathrm{Me}$, $\mathrm{NO}_{2}$ and isopropyl groups. Also, this procedure was equally effective for thiourea.

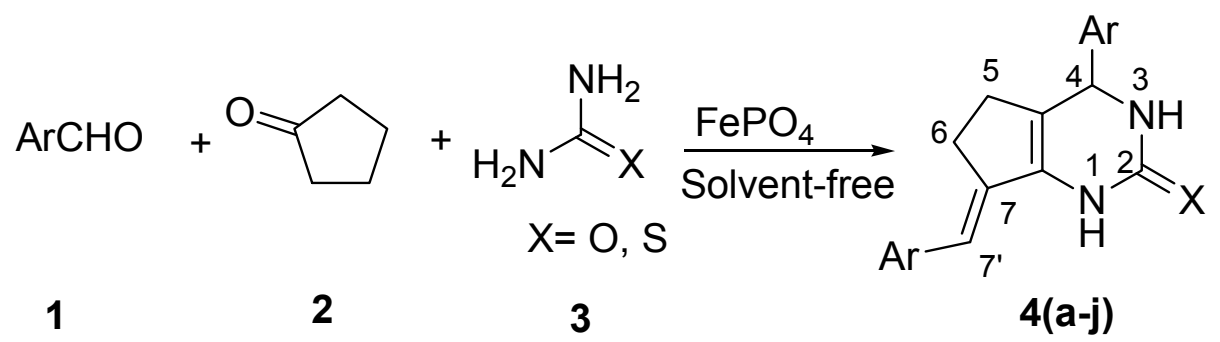

Scheme 1

Table 1. Synthesis of arylidenepyrimidinones and thiones using $\mathrm{FePO}_{4}$

\begin{tabular}{clccc}
\hline Product & $\mathbf{A r}$ & $\mathbf{X}$ & Time(h) & Yield\% \\
\hline $\mathbf{4 a}$ & $\mathrm{C}_{6} \mathrm{H}_{5-}$ & $\mathrm{O}$ & 4.0 & 90 \\
$\mathbf{4 b}$ & $4-\mathrm{Cl}_{-}-\mathrm{C}_{6} \mathrm{H}_{4}-$ & $\mathrm{O}$ & 4.5 & 80 \\
$\mathbf{4 c}$ & $3-\mathrm{NO}_{2}-\mathrm{C}_{6} \mathrm{H}_{4-}$ & $\mathrm{O}$ & 3.0 & 90 \\
$\mathbf{4 d}$ & $4-\mathrm{CH}_{3}-\mathrm{C}_{6} \mathrm{H}_{4-}$ & $\mathrm{O}$ & 6.0 & 80 \\
$\mathbf{4 e}$ & $\mathrm{C}_{6} \mathrm{H}_{5-}$ & $\mathrm{S}$ & 5.0 & 80 \\
$\mathbf{4 f}$ & $4-\mathrm{Cl}_{-}-\mathrm{C}_{6} \mathrm{H}_{4-}$ & $\mathrm{S}$ & 5.5 & 75 \\
$\mathbf{4 g}$ & $3-\mathrm{NO}_{2}-\mathrm{C}_{6} \mathrm{H}_{4}-$ & $\mathrm{S}$ & 4.5 & 80 \\
$\mathbf{4 h}$ & $4-\left(\mathrm{CH}_{3}\right)_{2} \mathrm{CH}_{-}-\mathrm{C}_{6} \mathrm{H}_{4}$ & $\mathrm{~S}$ & 5.0 & 75 \\
$\mathbf{4 i}$ & $4-\mathrm{NO}_{2}-\mathrm{C}_{6} \mathrm{H}_{4-}$ & $\mathrm{O}$ & 4.0 & 90 \\
$\mathbf{4 j}$ & $4-\left(\mathrm{CH}_{3}\right)_{2} \mathrm{~N}_{-}-\mathrm{C}_{6} \mathrm{H}_{4-}$ & $\mathrm{O}$ & 4.5 & 70 \\
\hline
\end{tabular}

The suggested mechanism ${ }^{9}$ of $\mathrm{FePO}_{4}$-catalyzed transformation is shown in Scheme 2. That involves formation of benzylidenecyclopentanone $\mathbf{1}$ and benzylideneurea $\mathbf{2}$ via aldol condensation aryl aldehyde with cyclopentanone and nucleophilic attacking of urea to $\mathrm{FePO}_{4}$-activated aldehyde. Coupling $\mathbf{1}$ and $\mathbf{2}$ following dehydration, leading to $\mathrm{FePO}_{4}$-activated intermediate 5 . The desired product 7 was resulted by ring closure of intermediate 5 following imine-enamine tautomerization 6 to 7 . 


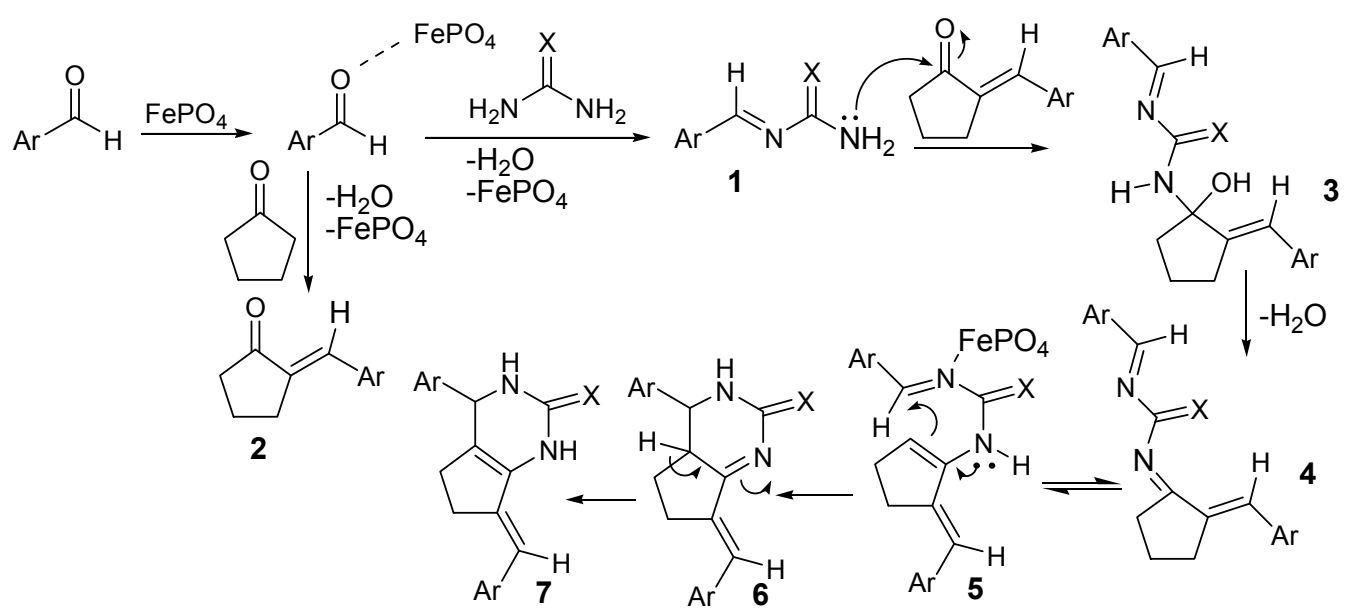

Scheme 2. Proposed mechanism for the synthesis of arylidenepyrimidinones and thiones using $\mathrm{FePO}_{4}$

To show advantages of this catalytic method with those of previously reported, the results of the formation of 7-benzylidene-4-phenyl-3,4,6,7-tetrahydro-1H-cyclopenta[d]pyrimidin-2(5H)-one (4a) were compared for a variety of catalysts (Table 2). From the results given in this Table 2, our method is evident, regarding the catalyst amounts, and high yield which are very important in chemical industry especially when it is combined with easy separation and reusability of the catalyst, and good yield.

Table 2. Synthesis of 7-benzylidene-4-phenyl-3,4,6,7-tetrahydro-1H-cyclopenta[d]pyrimidin$2(5 \mathrm{H})$-one catalyzed by various catalysts.

\begin{tabular}{|c|c|c|c|c|c|}
\hline Entry & Catalyst (mol\%) & Condition & Time (h) & Yield $\%$ & Ref. \\
\hline 1 & TMSCl (100) & DMF- $\mathrm{CH}_{3} \mathrm{CN} / \mathrm{rt}$ & 3.0 & 93 & 8 \\
\hline 2 & $\mathrm{YbCl}_{3}(3)$ & Neat $/ 90^{\circ} \mathrm{C}$ & 3.0 & 79 & 9 \\
\hline 3 & $\mathrm{IL}^{\mathrm{a}}(5)$ & Neat $/ 100^{\circ} \mathrm{C}$ & 0.1 & 86 & 12 \\
\hline 4 & {$[\mathrm{Hmim}] \mathrm{HSO}_{4}{ }^{\mathrm{b}}(5)$} & Solvent-free $/ 110^{\circ} \mathrm{C}$ & 1.0 & 38 & 13 \\
\hline 5 & {$\left[\mathrm{Hmim} \mathrm{HSO}_{4}(10)\right.$} & Solvent-free $/ 110^{\circ} \mathrm{C}$ & 1.0 & 52 & 9 \\
\hline 6 & {$[\mathrm{Hmim}] \mathrm{HSO}_{4}(15)$} & Solvent-free $/ 110^{\circ} \mathrm{C}$ & 0.75 & 65 & 9 \\
\hline 7 & {$[\mathrm{Hmim}] \mathrm{HSO}_{4}(25)$} & Solvent-free $/ 25^{\circ} \mathrm{C}$ & 1.5 & trace & 9 \\
\hline 8 & {$[\mathrm{Hmim}] \mathrm{HSO}_{4}(25)$} & Solvent-free $/ 80^{\circ} \mathrm{C}$ & 1.0 & 63 & 9 \\
\hline 9 & $\mathrm{FePO}_{4}(10)$ & Solvent-free $/ 110^{\circ} \mathrm{C}$ & 4.0 & 90 & This work \\
\hline
\end{tabular}

\section{Conclusions}

$\mathrm{FePO}_{4}$ was used as an inexpensive, easily available, non-corrosive and environmentally benign catalyst for the synthesis of arylidene heterobicyclicpyrimidinones by one-pot three component condensation reactions. Using solvent-free conditions, non-toxic and inexpensive materials, simple and clean work-up and high yields of the products are the advantages of this method. Three new derivatives of arylidene heterobicyclicpyrimidinones (entries 8-10; Table 1) were also synthesized by this new protocol.

\section{Experimental}

Melting points were measured by using the capillary tube method with an electro thermal 9200 apparatus. IR spectra were recorded on Perkin Elmer FT-IR spectrometer did scanning between 4000$400 \mathrm{~cm}^{-1}$. ${ }^{1} \mathrm{HNMR}$ and ${ }^{13} \mathrm{CNMR}$ spectra were obtained on Bruker DRX- $300 \mathrm{MHz}$ NMR instrument. Analytical TLC of all reactions was performed on Merck precoated plates (silica gel $60 \mathrm{~F}-254$ on aluminium). 
General procedure for the synthesis of arylidene heterobicyclicpyrimidinones using $\mathrm{FePO}_{4}$

A mixture of the aldehyde $(2.0 \mathrm{mmol}, \mathbf{4 a}, \mathbf{4 e} 0.212 \mathrm{~g} ; \mathbf{4 b}, \mathbf{4 f} 0.280 \mathrm{~g} ; \mathbf{4 c} 0.302 \mathrm{~g} ; \mathbf{4 d} 0.240 \mathrm{~g} ; \mathbf{4 h}$ $0.296 \mathrm{~g} ; 4 \mathbf{i} 0.302 \mathrm{~g} \mathrm{4j} 0.298 \mathrm{~g}$ ), cyclopentanone (1.1 mmol, $0.084 \mathrm{~g}$ ), urea or thiourea (1.2 mmol, 0.072 $\mathrm{g}$ or $0.0913 \mathrm{~g})$ and $\mathrm{FePO}_{4}(20 \mathrm{~mol} \%, 0.0302 \mathrm{~g})$ was heated in an oil bath at $110{ }^{\circ} \mathrm{C}$ for the specified times. The reaction was followed by TLC (ethyl acetate/cyclohexane, 50:50). After completion of the reaction, hot ethanol $(15 \mathrm{ml})$ was added and the catalyst was filtered off. Then the liquor was cooled to room temperature to form solid product. The solid product was collected by filtration, washed with water and then washed with ethanol to afford the pure product. The reusability of the catalyst was also studied. At the end of the reaction, the catalyst was filtered off, washed by ethanol or dichloromethane, and dried at $80^{\circ} \mathrm{C}$. Then the catalyst was subjected for three runs. After three runs, the catalytic activity of the catalyst was almost the same as those of the freshly used catalyst (Table 3 ).

Table 3. The reusability of the catalyst

\begin{tabular}{llll}
\hline Run & $\mathbf{1}$ & $\mathbf{2}$ & $\mathbf{3}$ \\
\hline Yield\% & 90 & 90 & 89 \\
\hline Reaction condition: Benzaldehyde $(2.0 \mathrm{mmol}, 0.212 \mathrm{~g})$, cyclopentanone $(1.1 \mathrm{mmol}, 0.092 \mathrm{~g})$, urea or thiourea $(1.2 \mathrm{mmol}, 0.072 \mathrm{~g} \mathrm{or} 0.0913 \mathrm{~g})$ and $\mathrm{FePO}_{4}$
\end{tabular}

$(20 \mathrm{~mol} \%, 0.0302 \mathrm{~g})$ under solvent-free condition at $110^{\circ} \mathrm{C}$.

Spectra and physical data for known products

7-(Benzylidene)-3,4,6,7-tetrahydro-4-phenyl-1H-cyclopentapyrimidin-2(5H)-one (4a)

White yellow solid; Yield\%: 90; m.p. 228-230 ${ }^{\circ} \mathrm{C}$, [236-239 ${ }^{\circ} \mathrm{C}$ (lit. 8)]; IR (KBr), $v$ cm$^{-1}: 3447$ (N-H), $3350(\mathrm{~N}-\mathrm{H}), 1682(\mathrm{C}=\mathrm{O}), 1599(\mathrm{C}=\mathrm{C}), 1464(\mathrm{C}=\mathrm{C}$, aromatic ring $) .{ }^{13} \mathrm{C}$ NMR $\left(\mathrm{DMSO}-\mathrm{d}_{6}\right): \delta 150.3(\mathrm{C} 2)$, 143.2 (C4'), 139.9 (C7), 135.2 (C7'), 128.7 ( Ar, =--NH), 127 (Ar), 126.4 (Ar), 124.5 (C7'; C=ㄷ), $115(=\underline{\mathrm{C}}), 59.8$ (C4), 28.1 (C5), $22(\mathrm{C} 6) ;{ }^{1} \mathrm{H}$ NMR (DMSO-d $): \delta 10.8$ (s, 1H, NH), $8.75(\mathrm{~s}, 1 \mathrm{H}, \mathrm{NH})$, 7.13-7.90 (m, $10 \mathrm{H}, \mathrm{Ar}-\mathrm{H}), 6.62(\mathrm{~s}, 1 \mathrm{H},=\mathrm{C} \underline{\mathrm{H}}), 5.30(\mathrm{~s}, 1 \mathrm{H}, \mathrm{C} 4 \mathrm{H})$ [5.15 (s, 1H, C4 H, Lit. 8], 2.22-2.10 (m, 2H, C9 H), 2.09-2.06 (m, 2H, C8 H).

7-(4-Chlorobenzylidene)-4-(4-chlorophenyl)-3,4,6,7-tetrahydro-1H-cyclopenta[d] pyrimidin-2(5H)one (4b)

White solid; Yield\%: 80; m.p. 249-253 ${ }^{\circ} \mathrm{C}$ [252-255 ${ }^{\circ} \mathrm{C}$ (lit. 8)]; IR (KBr), $v$ cm ${ }^{-1}: 3338(\mathrm{~N}-\mathrm{H}), 1681$ $(\mathrm{C}=\mathrm{O}), 1592(\mathrm{C}=\mathrm{C}), 1489\left(\mathrm{C}=\mathrm{C}\right.$, aromatic ring). ${ }^{13} \mathrm{C}$ NMR (DMSO-d $\left.{ }_{6}\right): \delta 150.3(\mathrm{C} 2), 141.3\left(\mathrm{C} 4{ }^{\prime}\right)$, 139.9 (C7), 133.3 (C7”), 132.3 ( C-Cl), 128.3 ( Ar, =C-NH), 127.6 (Ar), 124.5 (C7'; C=CH), 115.5 $(=\mathrm{C}), 59.8(\mathrm{C} 4), 28.1(\mathrm{C} 5), 22(\mathrm{C} 6) ;{ }^{1} \mathrm{H}$ NMR $\left(\mathrm{DMSO}_{-} \mathrm{d}_{6}\right): \delta 9.91(\mathrm{~s}, 1 \mathrm{H}, \mathrm{NH}), 8.91(\mathrm{~s}, 1 \mathrm{H}, \mathrm{NH}), 7.52-$ $7.9(\mathrm{~m}, 8 \mathrm{H}, \mathrm{Ar}-\mathrm{H}), 6.62(\mathrm{~s}, 1 \mathrm{H},=\mathrm{C} \underline{\mathrm{H}}), 5.18(\mathrm{~s}, 1 \mathrm{H}, \mathrm{C} 4 \mathrm{H})$ [5.18 (s, 1H, C4 H, Lit. 8], 2.85-2.73 (m, 2 H, C9 H), 2.41-2.36 (m, 2 H, C8 H).

7-(3-nitrobenzylidene)-3,4,6,7-tetrahydro-4-(3-nitrophenyl)-1H-cyclopentapyrimidin-2(5H)-one (4c) White yellow solid; Yield\%: 90; m.p. $234-238^{\circ} \mathrm{C}$ [235-239 ${ }^{\circ} \mathrm{C}$ (lit. 8)]; IR (KBr), $v$ cm$^{-1}: 3302(\mathrm{~N}-\mathrm{H})$, $1665(\mathrm{C}=\mathrm{O}), 1615(\mathrm{C}=\mathrm{C}), 1531\left(\mathrm{C}=\mathrm{C}\right.$, aromatic ring). ${ }^{13} \mathrm{C}$ NMR (DMSO-d $)$ ): $\delta 150.3(\mathrm{C} 2), 148.2(\underline{\mathrm{C}}-$ $\mathrm{NO}_{2}$ ), 144.1 (C4'), 139.9 (C7), 136.1 (C7'), 132.5 (Ar), 129.6 ( Ar) 128.3 ( =C- $\left.-\mathrm{NH}\right), 124.5$ (C7'; $\mathrm{C}=\underline{\mathrm{C}} \mathrm{H}$ ), $122.2(\mathrm{Ar}), 121.3$ (Ar), 120.3 (Ar) $115.5(=\underline{\mathrm{C}}), 58.8$ (C4), 28.1 (C5), 22 (C6); ${ }^{1} \mathrm{HNMR}$ (DMSO-d $)_{6}: \delta 10.13(\mathrm{~s}, 1 \mathrm{H}, \mathrm{NH}), 8.85(\mathrm{~s}, 1 \mathrm{H}, \mathrm{NH}), 8.68-8.33(\mathrm{~m}, 4 \mathrm{H}, \mathrm{Ar}-\mathrm{H}), 8.31-8.11(\mathrm{~m}, 4 \mathrm{H}, \mathrm{Ar}-$ $\mathrm{H}), 6.89(\mathrm{~s}, 1 \mathrm{H},=\mathrm{CH}), 5.77(\mathrm{~s}, 1 \mathrm{H}, \mathrm{C} 4 \mathrm{H})[5.45(\mathrm{~s}, 1 \mathrm{H}, \mathrm{C} 4 \mathrm{H}$, Lit. 8], 2.41-2.36 (m, 2H, C9 H) 1.15$1.13(\mathrm{~m}, 2 \mathrm{H}, \mathrm{C} 8 \mathrm{H})$.

7-(4-methylbenzylidene)-3,4,6,7-tetrahydro-4-(4-methylphenyl)-1H-cyclopentapyrimidin-2(5H)-one $(4 d)$

White solid; Yield\%: 80; m.p. 235-2240 ${ }^{\circ} \mathrm{C}$ [238-241 ${ }^{\circ} \mathrm{C}$ (lit. 8)]; IR (KBr), $v$ cm$^{-1}: 3441$ (N-H), 3348 $(\mathrm{N}-\mathrm{H}), 1678(\mathrm{C}=\mathrm{O}), 1508(\mathrm{C}=\mathrm{C}), 1464\left(\mathrm{C}=\mathrm{C}\right.$, aromatic ring). ${ }^{13} \mathrm{C}$ NMR (DMSO-d $)$ : $\delta 150.3(\mathrm{C} 2)$,

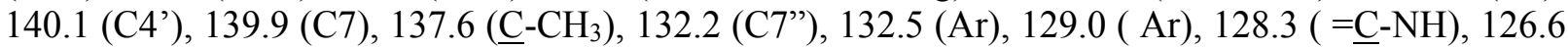


(Ar), 124.5 (C7'; C=대), 115.5 (=ㄷ), 59.8 (C4), 28.1 (C5), $24.3\left(\mathrm{CH}_{3}\right), 22$ (C6); ${ }^{1} \mathrm{H}$ NMR (DMSO$\left.\mathrm{d}_{6}\right): \delta 8.73(\mathrm{~s}, 1 \mathrm{H}, \mathrm{NH}), 7.23-7.14(\mathrm{~m}, 9 \mathrm{H}, \mathrm{Ar}-\mathrm{H}, \mathrm{NH}), 6.58(\mathrm{~s}, 1 \mathrm{H},=\mathrm{CH}), 5.09(\mathrm{~s}, 1 \mathrm{H}, \mathrm{C} 4 \mathrm{H})[5.09(\mathrm{~s}$, 1H, C4 H, Lit. 8], 2.82-2.71 (m, 2 H, C9 H), 2.38-2.33 (m, 2H, C8 H), 2.28 (s, 3H, C $\left.\mathrm{CH}_{3}\right)$.

7-Benzylidene-3,4,6,7-tetrahydro-4-phenyl-1H-cyclopentapyrimidine-2(5H)-thione (4e)

White yellow solid; Yield\%: 80; m.p. 215-220 ${ }^{\circ} \mathrm{C}$ [219-223 ${ }^{\circ} \mathrm{C}$ (lit. 8)]; IR (KBr), $v$ cm$^{-1}: 3380(\mathrm{~N}-\mathrm{H})$, $3168(\mathrm{~N}-\mathrm{H}), 1604(\mathrm{C}=\mathrm{S}), 1542(\mathrm{C}=\mathrm{C}), 1448(\mathrm{C}=\mathrm{C}$, aromatic ring $) .{ }^{13} \mathrm{C}$ NMR $\left(\mathrm{DMSO}-\mathrm{d}_{6}\right): \delta 174.5(\mathrm{C} 2)$, 143.2 (C4'), 141.3 ( = C-NH), 139.9 (C7), 135.2 (C7'), 129.0 ( Ar), 128.1 (Ar), 126.4 (Ar), 124.5 (C7'; $\mathrm{C}=\underline{\mathrm{CH}}), 113.3(=\mathrm{C}), 64.8(\mathrm{C} 4), 28.1(\mathrm{C} 5), 23.0(\mathrm{C} 6) ;{ }^{1} \mathrm{H}$ NMR $\left(\mathrm{DMSO}-\mathrm{d}_{6}\right): \delta 10.30(\mathrm{~s}, 1 \mathrm{H}, \mathrm{NH}), 9.18$ $(\mathrm{s}, 1 \mathrm{H}, \mathrm{NH}), 8.29-7.54(\mathrm{~m}, 10 \mathrm{H}, \mathrm{Ar}-\mathrm{H}), 7.09(\mathrm{~s}, 1 \mathrm{H},=\mathrm{CH}), 5.35(\mathrm{~s}, 1 \mathrm{H}, \mathrm{C} 4 \mathrm{H})[5.09(\mathrm{~s}, 1 \mathrm{H}, \mathrm{C} 4 \mathrm{H}$, Lit. 8], 2.93-2.86 (m, 2H, C9 H), 2.52-2.49 (m, 2H, C8 H ).

7-(4-chlorobenzylidene)-4-(4-chlorophenyl)- 3,4,6,7-tetrahydro-1H-cyclopentapyrimidine-2(5H)thione (4f)

White solid; Yield\%: 75; m.p. 219-224 ${ }^{\circ} \mathrm{C}$ [226-228 ${ }^{\circ} \mathrm{C}$ (lit. 8)]; IR (KBr), $v$ cm$^{-1}$ : 3441 (N-H), 3345 $\left.(\mathrm{N}-\mathrm{H}), 1660(\mathrm{C}=\mathrm{O}), 1602(\mathrm{C}=\mathrm{C}), 1547\left(\mathrm{C}=\mathrm{C} \text {, aromatic ring). }{ }^{13} \mathrm{C} \text { NMR (DMSO-d }\right)_{6}\right): \delta 174.5(\mathrm{C} 2)$, 141.3 (C4', =ㄷ-NH ), 139.9 (C7), 133.2 (C7”), 132.3 (C-Cl), 128.7 ( Ar), 128.4 (Ar), 126.4 (Ar), 124.5 $\left(\mathrm{C}^{\prime}\right.$; $\left.\mathrm{C}=\underline{\mathrm{CH}}\right), 113.3(=\mathrm{C}), 64.9$ (C4), 28.1 (C5), $23.0(\mathrm{C} 6) ;{ }^{1} \mathrm{H}$ NMR (DMSO-d 6$): \delta 10.2(\mathrm{~s}, 1 \mathrm{H}, \mathrm{NH})$, $8.69(\mathrm{~s}, 1 \mathrm{H}, \mathrm{NH}), 7.32-6.88(\mathrm{~m}, 8 \mathrm{H}, \mathrm{Ar}-\mathrm{H}), 6.98(\mathrm{~s}, 1 \mathrm{H},=\mathrm{CH}), 5.49(1 \mathrm{H}, \mathrm{s}, \mathrm{C} 4 \mathrm{H})[5.49(\mathrm{~s}, 1 \mathrm{H}, \mathrm{C} 4 \mathrm{H}$, Lit. 8], 2.73-2.68 (2H, m, C9 H) 2.40-2.36 (m, 2H, C8 H).

7-(4-nitrobenzylidene)-3,4,6,7-tetrahydro-4-(4-nitrophenyl)-1H-cyclopentapyrimidin-2(5H)-one (4i) White yellow solid; Yield\%: 90; m.p. 203-205 ${ }^{\circ} \mathrm{C}$, [204-207 ${ }^{\circ} \mathrm{C}$ (lit. 13)]; IR (KBr), $v \mathrm{~cm}^{-1}: 3449$ (N$\mathrm{H}), 3344(\mathrm{~N}-\mathrm{H}), 1667(\mathrm{C}=\mathrm{O}), 1519\left(\mathrm{NO}_{2}\right.$, asymmetry $), 1466(\mathrm{C}=\mathrm{C}), 1349\left(\mathrm{NO}_{2}\right.$, symmetry $) .{ }^{13} \mathrm{C} \mathrm{NMR}$ (DMSO-d $): \delta$ 150.3(C2), 149.3 (C4'), 147 (C4”), 146.0 (C4”), 141.0 (C6), 139.8(C7), 128.3 (Ar), 127.3 (Ar), $124.5(\mathrm{C}=\underline{\mathrm{C}} \mathrm{H}), 121.0(\mathrm{Ar}), 115.5$ (C5), 60.0 (C4), 28.1 (C8), 22.2 (C9); ${ }^{1} \mathrm{H}$ NMR (DMSO$\left.\mathrm{d}_{6}\right): \delta 10.15(\mathrm{~s}, 1 \mathrm{H}, \mathrm{NH}), 8.42(\mathrm{~s}, 1 \mathrm{H}, \mathrm{NH}), 7.51-8.39(\mathrm{~m}, 8 \mathrm{H}, \mathrm{Ar}-\mathrm{H}), 6.96(\mathrm{~s}, 1 \mathrm{H},=\mathrm{C} \underline{\mathrm{H}}), 5.76(\mathrm{~s}, 1 \mathrm{H}$, $\mathrm{C} 4 \mathrm{H}), 2.82-2.99$ (m, 2H, C9 H), 2.54-2.61 (2H, m, C8 H). [M+]: 392.11, Elemental analysis: Found, \%: C, 61.12; H, 4.09; N, 14.17. $\mathrm{C}_{20} \mathrm{H}_{16} \mathrm{~N}_{4} \mathrm{O}_{5}$. Calculated, \%: C, 61.22; H, 4.11; N, 14.28.

Spectra and physical data for unprecedented products

\section{7-(3-nitrobenzylidene)-3,4,6,7-tetrahydro-4-(3-nitrophenyl)-1H-cyclopentapyrimidine-2(5H)-thione (4g)}

White yellow solid; Yield\%: 90; m.p. 295-300 ${ }^{\circ} \mathrm{C}$; IR (KBr), $v \mathrm{~cm}^{-1}$ : $3292(\mathrm{~N}-\mathrm{H}), 1658(\mathrm{C}=\mathrm{O}), 1614$ $(\mathrm{C}=\mathrm{C}), 1530\left(\mathrm{NO}_{2}\right.$, asymmetry), $1503\left(\mathrm{C}=\mathrm{C}\right.$, aromatic ring) $1350\left(\mathrm{NO}_{2}\right.$, symmetry). ${ }^{13} \mathrm{C}$ NMR (DMSOd 6 ): $\delta 174.5(\mathrm{C} 2), 148.2\left(\underline{\mathrm{C}}-\mathrm{NO}_{2}\right), 144.1$ (C4'), 141.3 (C4'; =ㅡ-NH ), 139.9 (C7), 136.2 (C7'), 133.1 (Ar), 128.7 ( Ar), 129.4 (Ar), 124.5 (C7'; C=ㅡㅐ), 22.2 (Ar), 121.3, 120.3 (Ar), 113.3 (=ㄷ), 63.9 (C4), 28.1 (C5), 23.0 (C6); ${ }^{1} \mathrm{HNMR}\left(\mathrm{DMSO}-\mathrm{d}_{6}\right): \delta 10.27(\mathrm{~s}, 1 \mathrm{H}, \mathrm{NH}), 9.20(\mathrm{~s}, 1 \mathrm{H}, \mathrm{NH}), 8.24-8.20(\mathrm{~m}, 4 \mathrm{H}$, Ar-H), 7.82-7.63 (m, 4H, Ar-H), $7.02(\mathrm{~s}, 1 \mathrm{H},=\mathrm{C} \underline{\mathrm{H}}), 5.52(\mathrm{~s}, 1 \mathrm{H}, \mathrm{C} 4 \mathrm{H})$ [5.52 (s, 1H, C4 H, Lit. 8], , 2.97-2.88 (m, 2 H, C9 H), 2.57-2.51 (m, 2 H, C8 H).

7-(4-isopropylbenzylidene)-3,4,6,7-tetrahydro-4-(4-isopropylphenyl)-1H-cyclopenta[d]pyrimidine2(5H)-thione (4h)

White solid; Yield\%: 75; m.p. 218-220 ${ }^{\circ} \mathrm{C}$; IR (KBr), $v \mathrm{~cm}^{-1}$ : $3383(\mathrm{~N}-\mathrm{H}), 3172(\mathrm{~N}-\mathrm{H}), 1607(\mathrm{C}=\mathrm{S})$, $1542(\mathrm{C}=\mathrm{C}), 1462\left(\mathrm{C}=\mathrm{C}\right.$, aromatic ring). ${ }^{13} \mathrm{C}$ NMR $\left(\mathrm{DMSO}-\mathrm{d}_{6}\right): \delta 174.5 .3(\mathrm{C} 2), 147.3\left(\mathrm{C} 4{ }^{\prime}\right), 146.7$ (C4'), 141.0 (C6), 140.4 (C4), 139.8 (C7), 132.4 (Ar), 126.3 (Ar), 124.5 (C=대), 113.5 (C5), 65.0 (C4), $36.2\left[\underline{\mathrm{CH}}\left(\mathrm{CH}_{3}\right)_{2}\right], 28.2(\mathrm{C} 8), 23.4\left[\mathrm{CH}\left(\underline{\mathrm{CH}}_{3}\right)_{2}\right], 23.2(\mathrm{C} 9) ;{ }^{1} \mathrm{HNMR}\left(\mathrm{DMSO}-\mathrm{d}_{6}\right): \delta 10.31(1 \mathrm{H}, \mathrm{s}$, $\mathrm{NH}), 9.91(\mathrm{~s}, 1 \mathrm{H}, \mathrm{NH}), 7.30-7.36(\mathrm{~m}, 4 \mathrm{H}, \mathrm{Ar}-\mathrm{H}), 7.25-7.30(\mathrm{~m}, 4 \mathrm{H}, \mathrm{Ar}-\mathrm{H}), 7.18(\mathrm{~s}, 1 \mathrm{H},=\mathrm{CH}), 5.42$ $(\mathrm{s}, 1 \mathrm{H}, \mathrm{C} 4 \mathrm{H}), 3.11\left(\mathrm{~m}, 2 \mathrm{H},\left[\mathrm{C} \underline{\mathrm{H}}\left(\mathrm{CH}_{3}\right)_{2}\right]\right), 2.82-2.98(\mathrm{~m}, 2 \mathrm{H}, \mathrm{C} 9 \mathrm{H}), 2.53-2.60(2 \mathrm{H}, \mathrm{m}, \mathrm{C} 8 \mathrm{H}) .1 .17$ (m, $\left.12 \mathrm{H},\left[\mathrm{CH}\left(\mathrm{CH}_{3}\right)_{2}\right]\right) . \quad[\mathrm{M}+]:$ 402.21, Elemental analysis: Found, \%: $\mathrm{C}, 77.55 ; \mathrm{H}, 7.48 ; \mathrm{N}, 6.89 ; \mathrm{S}, 7.91$. $\mathrm{C}_{26} \mathrm{H}_{30} \mathrm{~N}_{2} \mathrm{~S}$. Calculated, \%: C, 77.57; H, 7.51; N, 6.96; S, 7.96 
7-(4-(dimethylaminobenzylidene)-4-(4-(dimethylamino)phenyl)-3,4,6,7-tetrahydro-1Hcyclopenta[d]pyrimidin-2(5H)-one $(4 j)$

White yellow solid; Yield\%: 90; m.p. 245-250 ${ }^{\circ} \mathrm{C}$; IR (KBr), $v \mathrm{~cm}^{-1}: 3350(\mathrm{~N}-\mathrm{H}), 3199(\mathrm{~N}-\mathrm{H}), 1664$ $(\mathrm{C}=\mathrm{O}), 1597(\mathrm{C}=\mathrm{C}), 1549\left(\mathrm{C}=\mathrm{C}\right.$, aromatic ring). ${ }^{13} \mathrm{C}$ NMR (DMSO- $\left.\mathrm{d}_{6}\right): \delta 150.3(\mathrm{C} 2), 148.3\left(\mathrm{C} 4^{\prime}\right), 147.6$ (C4”), 139.8(C7), 132.7(Ar), 128.3 (C6), 127.3 (Ar), $124.5(\mathrm{C}=\underline{\mathrm{CH}}), 115.5$ (C5), 114.2 (Ar), 59.8 (C4), $40.3\left(\mathrm{~N}\left(\underline{\mathrm{CH}}_{3}\right)_{2}\right), 28.1$ (C9), $22.2(\mathrm{C} 8) ;{ }^{1} \mathrm{H} \mathrm{NMR}$ (DMSO-d 6$): \delta 9.63(\mathrm{~s}, 1 \mathrm{H}, \mathrm{NH}), 9.20(\mathrm{~s}, 1 \mathrm{H}, \mathrm{NH})$, $\left.7.63(\mathrm{~m}, 4 \mathrm{H}, \mathrm{Ar}-\mathrm{H}), 7.65(\mathrm{~m}, 4 \mathrm{H}, \mathrm{Ar}-\mathrm{H}), 6.75(\mathrm{~s}, 1 \mathrm{H},=\mathrm{C} \underline{\mathrm{H}}), 5.35(\mathrm{~s}, 1 \mathrm{H}, \mathrm{C} 4 \mathrm{H}), 3.47\left(\mathrm{~s}, 6 \mathrm{H}, \mathrm{N}^{\left(\mathrm{CH}_{3}\right.}\right)_{2}\right)$, $3.41\left(\mathrm{~s}, 6 \mathrm{H}, \mathrm{N}\left(\mathrm{CH}_{3}\right)_{2}\right), 2.82-2.99(\mathrm{~m}, 2 \mathrm{H}, \mathrm{C} 9 \mathrm{H}), 2.54-2.61(\mathrm{~m}, 2 \mathrm{H}, \mathrm{C} 8 \mathrm{H})$. [M+]: 388.23, Elemental analysis: Found, \%: C, 74.15; H, 7.16; N, 14.38. $\mathrm{C}_{24} \mathrm{H}_{28} \mathrm{~N}_{4} \mathrm{O}$. Calculated, \%: C, 74.20; H, 7.26; N, 14.42 .

\section{References}

1. Atwal, K. S., Rovnyak, G.C., Reilly, B. C. Ó., and Schwartz, J. (1989) Substituted 1,4-dihydropyrimidines. III: Synthesis of selectively functionalized 2-hetero-1,4-dihydropyrimidines. J. Org. Chem., 54(25), 58985907.

2. Kappe, C.O., Fabian, W.M.F., and Semones, M.A. (1997) Conformational analysis of 4-aryldihydropyrimidine calcium channel modulators. A comparison of ab initio, semiempirical and X-ray crystallographic studies. Tetrahedron 53(8), 2803-2816.

3. Rashad, A. E., Shamroukh, A.H., Yousif, N. M., Salama, M.A.; Ali, H.S.; Ali, M. M., Mahmoud, A.E., and El-Shahat, M. (2012) New pyrimidinone and fused pyrimidinone derivatives as potential anticancer chemotherapeutics. Archiv der Pharmazie, 345(9), 729-738.

4. El-Subbagh, H. I., Abu-Zaid, S. M., Mahran, M. A., Badria, F. A., and Al-Obaid, A. M. (2000) Synthesis and biological evaluation of certain $\alpha, \beta$-unsaturated ketones and their corresponding fused pyridines as antiviral and cytotoxic agents. J. Med. Chem., 43(15), 2915-2921

5. Lorand, T., Deli, J., Szabo, D., Foeldesi, A., and Zschunke, A. (1985) Potentially bioactive pyrimidine derivatives. III: 4-Aryl-8-arylidene-6-methyl-3, 4, 5, 6, 7, 8-hexahydropyrido [4, 3-d] pyrimidine-2 [1H]ones and-2 (1H)-thiones. Pharmazie, 40(8), 536-539.

6. Elgemeie, G. E. H., Attia, A. M. E., and Alkabai, S. S. (2000) Nucleic acid components and their analogues: new synthesis of bicyclic thiopyrimidine nucleosides. Nucleos. Nucleot. Nucl, 19(4), 723-733.

7. Hammam, A. E. G., Sharaf, M. A., and El-Hafez, N. A. A. (2001) Synthesis and anti-cancer activity of pyridine and thiazolopyrimidine derivatives using 1-ethylpiperidone as a synthon. Indian J. Chem. Sec. B, 40(3), 213-221.

8. Zhu, Y., Huang, S., and Pan, Y. (2005) Highly chemoselective multicomponent biginelli-type condensations of cycloalkanones, urea or thiourea and aldehydes. Eur. J. Org. Chem., 2005(11), 2354-2367.

9. Zhang, H., Zhou, Z., Yao, Z., Xu, F., and Shen, Q. (2009) Efficient synthesis of pyrimidinone derivatives by ytterbium chloride catalyzed Biginelli-type reaction under solvent-free conditions. Tetrahedron Lett., 50(14), 1622-1624.

10. Behbahani, F. K., and Farahani, M. (2011) Anhydrous $\mathrm{FePO}_{4}$ as a cost-effective and recyclable catalyst for tetrahydropyranylation and tetrahydrofuranylation of alcohols and phenols. Lett. Org. Chem., 8(6), 431435.

11. Behbahani, F. K., and Yazdanparast, B. (2014) Iron (III) phosphate catalyzed synthesis of 1, 4dihydropyridines. Arabian J. Chem., inpress. doi.org/10.1016/j.arabjc.2014.11.027

12. Hajipour, A. R., Ghayeb,Y., Sheikhan, N., and Ruoho, A. E. (2011) Brønsted acidic ionic liquid as an efficient and reusable catalyst for one-pot, three-component synthesis of pyrimidinone derivatives via Biginelli-type reaction under solvent-free conditions. Synthetic commun., 41(15), 2226-2233.

13. Kiyani, H., and Ghiasi, M. (2014). Potassium phthalimide: An efficient and green organocatalyst for the synthesis of 4-aryl-7-(arylmethylene)-3, 4, 6, 7-tetrahydro-1H-cyclopenta [d] pyrimidin-2 (5H)ones/thiones under solvent-free conditions. Chin. Chem. Lett., 25(2), 313-316.

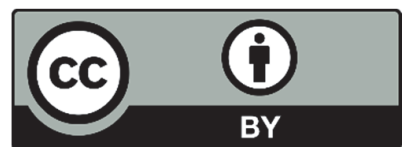

(C) 2018 by the authors; licensee Growing Science, Canada. This is an open access article distributed under the terms and conditions of the Creative Commons Attribution (CC-BY) license (http://creativecommons.org/licenses/by/4.0/). 